\title{
The Condition Number of the Schur Complement in Domain Decomposition *
}

\author{
Susanne C. Brenner \\ Department of Mathematics \\ University of South Carolina \\ Columbia, SC 29208
}

Dedicated to Olof B. Widlund on the occasion of his 60th birthday.

Summary. It is shown that for elliptic boundary value problems of order $2 m$ the condition number of the Schur complement matrix that appears in nonoverlapping domain decomposition methods is of order $d^{-1} h^{-2 m+1}$, where the parameter $d$ measures the diameters of the subdomains and $h$ is the mesh size of the triangulation. The result holds for both conforming and nonconforming finite elements.

Mathematics Subject Classification (1991): 65N55, 65N30.

\section{Introduction}

In the nonoverlapping approach to domain decomposition (cf. [10], [21] and the references therein) the finite element equation for an elliptic boundary value problem is reduced, after parallel subdomain solves, to its Schur complement which involves only the nodal variables (degrees of freedom) on the subdomain boundaries. The condition number of the Schur complement has been investigated for second order elliptic problems and conforming finite elements in [3] and [18], and for a finite difference scheme for the biharmonic equation on a rectangle with two subdomains in [11]. We present in this paper a general study of the condition number of the Schur complement in the context of finite element methods which covers elliptic problems of any order and both conforming and nonconforming finite elements.

Let $\Omega$ be a bounded open polyhedral domain $\Omega \subseteq \mathbb{R}^{n}(n=2$ or 3 ) and $a(\cdot, \cdot)$ (the variational form of the elliptic boundary value problem) be a symmetric bilinear form on $H^{m}(\Omega)$ ( $m$ is a positive integer) which is defined by an integral over $\Omega$. We assume that there exist positive constants $C_{1}$ and $C_{2}$ such that

$$
C_{1}\|v\|_{H^{m}(\Omega)}^{2} \leq a(v, v) \leq C_{2}\|v\|_{H^{m}(\Omega)}^{2} \quad \forall v \in V,
$$

where $V$ is a closed subspace of $H^{m}(\Omega)$ and $H_{0}^{m}(\Omega) \subseteq V$.

Let $\mathcal{T}_{h}$ be a quasi-uniform triangulation (cf. [12], [8]) of $\Omega$ and $h=\max _{T \in \mathcal{T}_{h}} \operatorname{diam} T$. Let $\Omega$ be subdivided into nonoverlapping polyhedral subdomains $\Omega_{j}(1 \leq j \leq J)$ whose

* This work was supported in part by the National Science Foundation under Grant No. DMS-96-00133. 
boundaries are aligned with $\mathcal{T}_{h}$ (cf. Figure 1 ). The union $\cup_{j=1}^{J} \partial \Omega_{j}$ of the subdomain boundaries will be denoted by $\Gamma$.

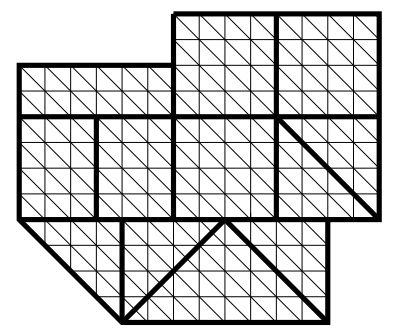

Figure 1

The boundaries of the subdomains consist of faces when $n=3$ and edges when $n=2$, and from here on we will refer to both as faces. Let $\mathcal{F}_{j}$ be the set of all the faces of $\Omega_{j}$. If $F \in \mathcal{F}_{j}$, then the trace map $\operatorname{Tr}_{F}: H^{m}\left(\Omega_{j}\right) \longrightarrow \Pi_{k=1}^{m} H^{m-k+(1 / 2)}(F)$ is defined by $\operatorname{Tr}_{F}(v)=\left(v, \partial_{n} v, \partial_{n}^{2} v, \ldots, \partial_{n}^{m-1} v\right)$, where $n$ is the unit outer normal of $F$.

Let $V_{h} \subseteq V$ be a (conforming) finite element space associated with $\mathcal{T}_{h}$ and $V_{h}(\Omega \backslash \Gamma)$ be the subspace of $v \in V_{h}$ such that $\operatorname{Tr}_{F}(v)=0$ for all $F \in \mathcal{F}_{j}$ and $1 \leq j \leq J$. The skeleton subspace $V_{h}(\Gamma) \subseteq V_{h}$ is defined to be the $a(\cdot, \cdot)$-orthogonal complement of $V_{h}(\Omega \backslash \Gamma)$.

Note that functions in $V_{h}(\Gamma)$ are completely determined by their traces on the subdomain faces. In fact, given any $u \in V_{h}$, the unique function $v \in V_{h}(\Gamma)$ that satisfies $\operatorname{Tr}_{F} v=\operatorname{Tr}_{F} u$ for all $F \in \mathcal{F}_{j}$ and $1 \leq j \leq J$ is determined by the conditions that $v-u \in V_{h}(\Omega \backslash \Gamma)$ and $a(v-u, w)=-a(u, w)$ for all $w \in V_{h}(\Omega \backslash \Gamma)$. We can therefore define an inner product $(\cdot, \cdot)_{h, \Gamma}$ on $V_{h}(\Gamma)$ by

$$
\left(v_{1}, v_{2}\right)_{h, \Gamma}=\sum_{j=1}^{J} \sum_{F \in \mathcal{F}_{j}} \sum_{\ell=0}^{m-1} h^{2 \ell}\left(\partial_{n}^{\ell} v_{1}, \partial_{n}^{\ell} v_{2}\right)_{L^{2}(F)}
$$

The Schur complement operator $\mathbf{S}_{h}: V_{h}(\Gamma) \longrightarrow V_{h}(\Gamma)$ is then defined by

$$
\left(\mathbf{S}_{h} v_{1}, v_{2}\right)_{h, \Gamma}=a\left(v_{1}, v_{2}\right) \quad \forall v_{1}, v_{2} \in V_{h}(\Gamma)
$$

Clearly $\mathbf{S}_{h}$ is a symmetric positive definite operator. The condition number of $\mathbf{S}_{h}$ is $\kappa\left(\mathbf{S}_{h}\right)=\lambda_{\max }\left(\mathbf{S}_{h}\right) / \lambda_{\min }\left(\mathbf{S}_{h}\right)$.

The main result of this paper states that, under certain assumptions on the subdomains $\Omega_{j}$ and the finite element space $V_{h}$, the following estimates hold:

$$
C_{3} d^{-1} h^{-2 m+1} \leq \kappa\left(\mathbf{S}_{h}\right) \leq C_{4} d^{-1} h^{-2 m+1}
$$

where $d=\max _{1 \leq j \leq J} \operatorname{diam} \Omega_{j}$, and the positive constants $C_{3}$ and $C_{4}$ are independent of $h, d$ and $J$.

In order to avoid the proliferation of constants, we will use from here on the notation $A \lesssim B$ (or $B \gtrsim A$ ) to represent the statement that $A \leq$ Constant $\times B$, where the constant is independent of $h, d, J$ and the variables in $A$ and $B$. The notation $A \approx B$ means that $A \lesssim B$ and $A \gtrsim B$. 
Let us examine a few examples at this point to connect $\kappa\left(\mathbf{S}_{h}\right)$ to the condition number of the usual Schur complement matrix. The descriptions of finite elements below will follow the conventions in [12] and [8].

Example 1.1. The variational form $a(\cdot, \cdot)$ for the Poisson equation with homogeneous Dirichlet boundary condition is defined by

$$
a\left(v_{1}, v_{2}\right)=\int_{\Omega} \nabla v_{1} \cdot \nabla v_{2} d x \quad \forall v_{1}, v_{2} \in H_{0}^{1}(\Omega)
$$

Some commonly used conforming finite elements for this equation are the $\mathcal{Q}_{1}$ and $\mathcal{P}_{1}$ elements for $n=2$, and the $\mathcal{Q}_{1}$ element for $n=3$ (cf. Figure 2, Figure 3 and Figure 4).

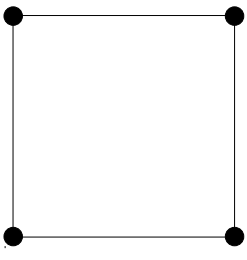

Figure 2

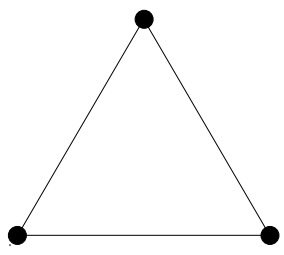

Figure 3

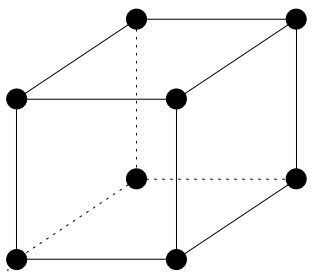

Figure 4

Let $V_{h}$ be the corresponding finite element space associated with $\mathcal{T}_{h}, N=\operatorname{dim} V_{h}$ and $p_{j}, 1 \leq j \leq N$, be the vertices inside $\Omega$. Let $\phi_{j}$ be defined by $\phi_{p_{j}}(p)=h^{-(n / 2)}$ when $p=p_{j}$ and 0 for any other vertices of $\mathcal{T}_{h}$. Then $\left\{\phi_{j}\right\}_{j=1}^{N}$ is a basis of $V_{h}$ and we have

$$
\|v\|_{L^{2}(\Omega)}^{2} \approx \sum_{j=1}^{N} x_{j}^{2} \quad \text { for } \quad v=\sum_{j=1}^{N} x_{j} \phi_{j}
$$

The components of the stiffness matrix $A$ are given by

$$
a_{i j}=a\left(\phi_{i}, \phi_{j}\right) \quad \text { for } \quad 1 \leq i, j \leq N
$$

Let $I$ be the index set of the vertices not in $\Gamma$ (the internal nodes), and $G$ be the index set of the vertices on $\Gamma$ (the skeletal nodes). Note that

$$
\sum_{j=1}^{J} \sum_{F \in \mathcal{F}_{j}}(v, v)_{L^{2}(F)} \approx h^{-1} \sum_{g \in G} x_{g}^{2} \quad \text { for } \quad v=\sum_{j=1}^{N} x_{j} \phi_{j}
$$

We can arrange the ordering of the $p_{j}$ 's so that the internal nodes come first, i.e., $I=$ $\left\{1,2, \ldots, n_{I}\right\}$ and $G=\left\{n_{I}+1, n_{I}+2, \ldots, n_{I}+n_{G}\right\}$ where $n_{I}$ and $n_{G}$ are the number of internal nodes and skeletal nodes respectively. The stiffness matrix $A$ can be written as

$$
A=\left[\begin{array}{cc}
A_{I I} & A_{I G} \\
A_{G I} & A_{G G}
\end{array}\right]
$$


where $A_{I I}, A_{G G}$ and $A_{I G}=A_{G I}^{t}$ are the submatrices representing the interaction among the basis functions corresponding to the two types of nodes. The Schur complement $S$ of the stiffness matrix $A$ is the matrix defined by

$$
S=A_{G G}-A_{G I} A_{I I}^{-1} A_{I G} .
$$

A function $v=\sum_{i \in I} x_{i} \phi_{i}+\sum_{g \in G} x_{g} \phi_{g}$ belongs to $V_{h}(\Gamma)$ if and only if $A_{I I} x^{I}+$ $A_{I G} x^{G}=0$, where $x^{I}=\left(x_{1}, x_{2}, \ldots, x_{n_{I}}\right)^{t}$ and $x^{G}=\left(x_{n_{I}+1}, x_{n_{I}+2}, \ldots, x_{n_{I}+n_{G}}\right)^{t}$. We can therefore define, for $\ell \in G$, a function $\tilde{\phi}_{\ell} \in V_{h}(\Gamma)$ as follows:

$$
\tilde{\phi}_{\ell}=\sum_{i \in I} x_{i, \ell} \phi_{i}+\sum_{g \in G} x_{g, \ell} \phi_{g}
$$

where $x_{g, \ell}=1$ for $g=\ell$ and 0 otherwise, and the coordinates $x_{i, \ell}$ are determined by

$$
A_{I I} x_{\ell}^{I}+A_{I G} x_{\ell}^{G}=0 .
$$

Then $\left\{\tilde{\phi}_{g}: g \in G\right\}$ is a basis of $V_{h}(\Gamma)$. Moreover it follows from (1.2) and (1.6) that

$$
\left(\sum_{g \in G} y_{g} \tilde{\phi}_{g}, \sum_{g \in G} y_{g} \tilde{\phi}_{g}\right)_{h, \Gamma} \approx h^{-1} \sum_{g \in G} y_{g}^{2} .
$$

In view of (1.5) and (1.7)-(1.10), the components $S_{i j}$ of $S$ are given by

$$
S_{i j}=a\left(\tilde{\phi}_{n_{I}+i}, \tilde{\phi}_{n_{I}+j}\right) \quad \text { for } \quad 1 \leq i, j \leq n_{G} .
$$

Let $v=\sum_{j=1}^{n_{G}} y_{j} \tilde{\phi}_{n_{I}+j}$. We have, by (1.3), (1.11) and (1.12),

$$
\frac{\left(\mathbf{S}_{h} v, v\right)_{h, \Gamma}}{(v, v)_{h, \Gamma}} \approx h \frac{y^{t} S y}{y^{t} y},
$$

where $y=\left(y_{1}, y_{2}, \ldots, y_{n_{G}}\right)^{t}$. The relation (1.13) among the Rayleigh quotients implies that

$$
\kappa\left(\mathbf{S}_{h}\right) \approx \kappa(S) .
$$

Example 1.2. The variational form for the biharmonic equation on $\Omega \subseteq \mathbb{R}^{2}$ with homogeneous Dirichlet boundary condition is defined by

$$
a\left(v_{1}, v_{2}\right)=\int_{\Omega} \sum_{i, j=1}^{2}\left(v_{1}\right)_{x_{i} x_{j}}\left(v_{2}\right)_{x_{i} x_{j}} d x \quad \forall v_{1}, v_{2} \in H_{0}^{2}(\Omega) .
$$

Some commonly used conforming finite elements for this equation are the Bogner-FoxSchmit element (cf. [4] and Figure 5), the Hsieh-Clough-Tocher macro element (cf. [15] and Figure 6) and the fifth degree Argyris element (cf. [2] and Figure 7). 


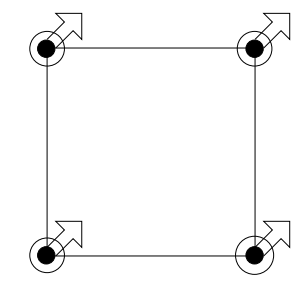

Figure 5

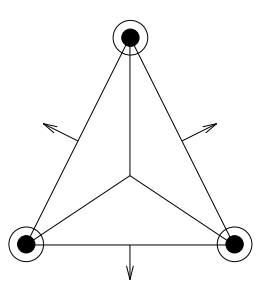

Figure 6

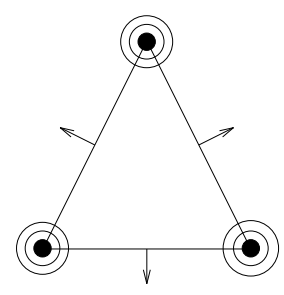

Figure 7

Let $V_{h}$ be the corresponding finite element space associated with $\mathcal{T}_{h}, N=\operatorname{dim} V_{h}$ and $\left\{N_{j}\right\}_{j=1}^{N}$ be the set of all nodal variables for $V_{h}$. For a nodal variable $N_{i}$ that evaluates a derivative of order $k$ we choose the corresponding nodal basis function $\phi_{i}$ so that $N_{i}\left(\phi_{i}\right)=$ $h^{-k-1}$ and $N_{j}\left(\phi_{i}\right)=0$ for $j \neq i$. Note that with this choice of the $\phi_{i}$ 's we have $\|v\|_{L^{2}(\Omega)}^{2} \approx$ $\sum_{j=1}^{N} x_{j}^{2}$ and $\sum_{j=1}^{J} \sum_{F \in \mathcal{F}_{j}} \sum_{\ell=0,1} h^{2 \ell}\left(\partial_{n}^{\ell} v, \partial_{n}^{\ell} v\right)_{L^{2}(F)} \approx h^{-1} \sum_{g \in G} x_{g}^{2}$ for $v=\sum_{j=1}^{N} x_{j} \phi_{j}$, where $G$ (respectively $I$ ) is the index set for the internal (respectively skeletal) nodal variables. The stiffness matrix $A=\left(a\left(\phi_{i}, \phi_{j}\right)\right)_{i, j=1}^{N}$ can be written in the form of (1.7), and its Schur complement $S$ is defined by (1.8). A completely analogous argument shows that (1.14) also holds here.

Remark 1.3. For simplicity we take $V$ to be $H_{0}^{m}(\Omega)$ in Examples 1.2 and 1.3. But the assumption (1.1) on the variational form and hence the results of this paper are valid for many other boundary conditions.

The rest of the paper is organized as follows. The precise assumptions on the subdomains and the conforming finite elements are given in Section 2. The condition number estimates for the Schur complements are established for conforming finite elements in Section 3 and extended to nonconforming finite elements in Section 4.

\section{Assumptions on the Subdomains and the Conforming Finite Element Space}

We assume $J \geq 2$ (so that $\Gamma \cap \Omega \neq \emptyset$ ) and that there are a fixed number of reference polyhedral domains $D_{k}(1 \leq k \leq K)$, each with unit diameter, such that for each $j$ there is an invertible affine map $\mu_{j}$ which maps $\Omega_{j}$ to one of the reference domains $D_{k(j)}$. Let $\mu_{j}(x)=\mathbf{A}_{j} x+b_{j}$ where $\mathbf{A}_{j}$ is a nonsingular $n \times n$ matrix. We assume that

$$
\left\|\mathbf{A}_{j}\right\|_{\infty} \lesssim d^{-1} \quad \text { and } \quad\left\|\mathbf{A}_{j}^{-1}\right\|_{\infty} \lesssim d \quad \text { for } \quad 1 \leq j \leq J
$$

Note that in particular $\operatorname{diam} \Omega_{j} \approx d$ and we do not assume that the subdomains $\Omega_{j}$ form a triangulation of $\Omega$ (cf. Figure 1$)$.

We can state the trace theorem (cf. [17]) on the reference domains as follows.

Lemma 2.1. Let $D$ be one of the reference domains. For any $\hat{v} \in H^{m}(D)$, we have $\left.\partial^{\alpha} \hat{v}\right|_{\partial D} \in H^{1 / 2}(\partial D)$ for $|\alpha| \leq m-1$ and $\sum_{|\alpha| \leq m-1}\left\|\partial^{\alpha} \hat{v}\right\|_{H^{1 / 2}(\partial D)} \lesssim\|\hat{v}\|_{H^{m}(D)}$. Moreover, there exists $\hat{\zeta} \in H^{m}(D)$ such that $\|\hat{\zeta}\|_{H^{m}(D)} \lesssim \sum_{|\alpha|<m-1}\left\|\partial^{\alpha} \hat{v}\right\|_{H^{1 / 2}(\partial D)}$ and $\operatorname{Tr}_{F} \hat{\zeta}=\operatorname{Tr}_{F} \hat{v}$ for all $F \in \mathscr{F}_{D}$, where $\mathscr{F}_{D}$ is the collection of all the faces of $D$. 
Combining Lemma 2.1 with the Bramble-Hilbert lemma (cf. [5]) we immediately obtain the estimate

$$
\left|\partial^{\alpha} \hat{v}\right|_{H^{1 / 2}(\partial D)} \lesssim|\hat{v}|_{H^{m}(D)} \quad \forall \hat{v} \in H^{m}(D) \quad \text { and } \quad|\alpha|=m-1
$$

Lemma 2.1, (2.2) and a scaling argument using (2.1) then gives the following trace theorem on the subdomains $\Omega_{j}$.

Lemma 2.2. Let $v_{j} \in H^{m}\left(\Omega_{j}\right)$. Then we have $\left.\partial^{\alpha} v_{j}\right|_{\partial \Omega_{j}} \in H^{1 / 2}\left(\partial \Omega_{j}\right)$ for $|\alpha| \leq m-1$,

$$
\begin{aligned}
\sum_{|\alpha| \leq m-1} d^{|\alpha|}\left[\left\|\partial^{\alpha} v_{j}\right\|_{L^{2}\left(\partial \Omega_{j}\right)}+d^{1 / 2}\left|\partial^{\alpha} v_{j}\right|_{H^{1 / 2}\left(\partial \Omega_{j}\right)}\right] & \lesssim \sum_{|\alpha| \leq m} d^{|\alpha|-(1 / 2)}\left\|\partial^{\alpha} v_{j}\right\|_{L^{2}\left(\Omega_{j}\right)} \\
\left|\partial^{\alpha} v_{j}\right|_{H^{1 / 2}\left(\partial \Omega_{j}\right)} & \lesssim\left|v_{j}\right|_{H^{m}\left(\Omega_{j}\right)} \quad \text { for } \quad|\alpha|=m-1 .
\end{aligned}
$$

Moreover, there exists $\zeta_{j} \in H^{m}\left(\Omega_{j}\right)$ such that

$$
\begin{aligned}
\sum_{|\alpha| \leq m} d^{|\alpha|-(1 / 2)}\left\|\partial^{\alpha} \zeta_{j}\right\|_{L^{2}\left(\Omega_{j}\right)} & \lesssim \sum_{|\alpha| \leq m-1} d^{|\alpha|}\left[\left\|\partial^{\alpha} v_{j}\right\|_{L^{2}\left(\partial \Omega_{j}\right)}+d^{1 / 2}\left|\partial^{\alpha} v_{j}\right|_{H^{1 / 2}\left(\partial \Omega_{j}\right)}\right] \\
\operatorname{Tr}_{F} \zeta_{j} & =\operatorname{Tr}_{F} v_{j} \quad \forall F \in \mathcal{F}_{j}
\end{aligned}
$$

The following are the assumptions on $V_{h}$.

\section{Interpolation Estimates}

There exists an interpolation operator $\Pi_{h}: V \longrightarrow V_{h}$ such that

$$
\left|\zeta-\Pi_{h} \zeta\right|_{H^{\ell}(\Omega)} \lesssim h^{m-\ell}|\zeta|_{H^{m}(\Omega)} \quad \forall \zeta \in V \quad \text { and } \quad 0 \leq \ell \leq m
$$

Moreover, $\Pi_{h}$ has the property that for any $F \in \mathcal{F}_{j}$

$$
\operatorname{Tr}_{F}\left(\Pi_{h} \zeta\right)=\operatorname{Tr}_{F}(\zeta) \text { if } \operatorname{Tr}_{F}(\zeta)=\operatorname{Tr}_{F}(v) \text { for some } v \in V_{h}
$$

Inverse Estimates

$$
\left|\partial^{\alpha} v\right|_{H^{1 / 2}(F)} \lesssim h^{-1 / 2}\left\|\partial^{\alpha} v\right\|_{L^{2}(F)} \quad \forall v \in V_{h},|\alpha| \leq m-1, F \in \mathcal{F}_{j} \text { and } 1 \leq j \leq J
$$

Nodal Variables of Order $m-1$

(2.10) The evaluations of all derivatives up to order $m-1$ at the vertices are nodal variables of the finite element. 
Remark 2.3. Since $V_{h} \subseteq H^{m}(\Omega)$, assumption (2.10) is natural. The estimate (2.9) follows from standard inverse estimates (cf. [12], [8]) and interpolation between Sobolev spaces (cf. [22]). The estimate (2.7) means that polynomials of degree $\leq m-1$ are shape functions of the finite element. The construction of interpolation operators that satisfy (2.7)-(2.8) can be found in [14] and [20]. In particular all of these assumptions are satisfied by the finite elements in Example 1.1 and Example 1.2.

\section{The Condition Number Estimates for the Schur Complement}

We begin with the estimate of the smallest eigenvalue of $\mathbf{S}_{h}$.

Lemma 3.1. The following estimate holds.

$$
d(v, v)_{h, \Gamma} \lesssim a(v, v) \quad \forall v \in V_{h}(\Gamma) .
$$

Proof. From (2.3) we find

$$
d \sum_{j=1}^{J} \sum_{F \in \mathcal{F}_{j}} \sum_{\ell=0}^{m-1} d^{2 \ell}\left\|\partial_{n}^{\ell} v\right\|_{L^{2}(F)}^{2} \lesssim \sum_{j=1}^{J} \sum_{k=0}^{m} d^{2 k}|v|_{H^{k}\left(\Omega_{j}\right)}^{2} \quad \forall v \in V_{h}(\Gamma) .
$$

Since $h \lesssim d$ and $d \leq \operatorname{diam} \Omega$, we deduce from (1.2) and (3.2) that

$$
d(v, v)_{h, \Gamma} \lesssim d \sum_{j=1}^{J} \sum_{F \in \mathcal{F}_{j}} \sum_{\ell=0}^{m-1} d^{2 \ell}\left\|\partial_{n}^{\ell} v\right\|_{L^{2}(F)}^{2} \lesssim \sum_{j=1}^{J} \sum_{k=0}^{m}|v|_{H^{k}\left(\Omega_{j}\right)}^{2} .
$$

The estimate (3.1) follows from (1.1) and (3.3).

Lemma 3.2. If $v \in V_{h}(\Gamma), \tilde{v} \in V_{h}$ and $\operatorname{Tr}_{F} v=\operatorname{Tr}_{F} \tilde{v}$ for all $F \in \mathcal{F}_{j}$ and $1 \leq j \leq J$, then $a(v, v) \leq a(\tilde{v}, \tilde{v})$.

Proof. Since $\tilde{v}-v \in V_{h}(\Omega \backslash \Gamma)$, it follows from the definition of $V_{h}(\Gamma)$ that

$$
a(\tilde{v}, \tilde{v})=a((\tilde{v}-v)+v,(\tilde{v}-v)+v)=a(\tilde{v}-v, \tilde{v}-v)+a(v, v) .
$$

The following lemma shows that (3.1) is the best possible estimate.

Lemma 3.3. There exists $v_{*} \in V_{h}(\Gamma)$ such that

$$
a\left(v_{*}, v_{*}\right) \lesssim d\left(v_{*}, v_{*}\right)_{h, \Gamma} .
$$

Proof. For $\epsilon>0$ we define $\Omega_{\epsilon}=\{x \in \Omega$ : $\operatorname{dist}(x, \partial \Omega)>\epsilon\}$ and $\Gamma_{\epsilon}=\Gamma \cap \Omega_{\epsilon}$. We can choose $\epsilon$ to be so small that

$$
\left|\Gamma_{2 \epsilon}\right| \gtrsim d^{-1}
$$


for subdomains that satisfy the assumption (2.1), where $\left|\Gamma_{2 \epsilon}\right|$ is the $(n-1)$-dimensional Lebesgue measure of $\Gamma_{2 \epsilon}$.

Let $f$ be a $C^{\infty}$ function supported in a compact subset of $\Omega$ such that

$$
f=1 \text { on } \Omega_{\epsilon} .
$$

Let $\tilde{v}=\Pi_{h} f$ and $v_{*} \in V_{h}(\Gamma)$ be defined by

$$
\operatorname{Tr}_{F} v_{*}=\operatorname{Tr}_{F} \tilde{v} \quad \forall F \in \mathcal{F}_{j}, 1 \leq j \leq J .
$$

It follows from (1.1), (2.7), (3.7) and Lemma 3.2 that

$$
a\left(v_{*}, v_{*}\right) \leq a(\tilde{v}, \tilde{v}) \lesssim\|f\|_{H^{m}(\Omega)}^{2} .
$$

On the other hand, condition (3.6) implies that $\tilde{v}=1$ on all the elements that are inside $\Omega_{\epsilon}$ (cf. Remark 2.3). We may also assume $h$ to be less than $\epsilon$, otherwise the lemma follows from Lemma 3.5 below. Hence $v_{*}=\tilde{v}=1$ on $\Gamma_{2 \epsilon}$. We deduce from (1.2), (3.5) and (3.7) that

$$
\left(v_{*}, v_{*}\right)_{h, \Gamma} \geq\left(v_{*}, v_{*}\right)_{L^{2}(\Gamma)} \geq\left(v_{*}, v_{*}\right)_{L^{2}\left(\Gamma_{2 \epsilon}\right)} \gtrsim d^{-1} .
$$

The estimate (3.4) follows from (3.8) and (3.9).

Corollary 3.4. We have $\lambda_{\min }\left(\mathbf{S}_{h}\right) \approx d$.

Proof. This is an immediate consequence of (1.3), (3.1), (3.4) and the Rayleigh quotient formula (cf. [13])

$$
\lambda_{\min }\left(\mathbf{S}_{h}\right)=\min _{\substack{v \in V_{h}(\Gamma) \\ v \neq 0}} \frac{\left(\mathbf{S}_{h} v, v\right)_{h, \Gamma}}{(v, v)_{h, \Gamma}} .
$$

Next we estimate the largest eigenvalue of $\mathbf{S}_{h}$.

Lemma 3.5. The following estimate holds.

$$
a(v, v) \lesssim h^{-2 m+1}(v, v)_{h, \Gamma} \quad \forall v \in V_{h}(\Gamma) .
$$

Proof. Let $v \in V_{h}(\Gamma)$ be arbitrary. By Lemma 2.2 there exists $\zeta_{j} \in H^{m}\left(\Omega_{j}\right)$ such that (2.5) and (2.6) hold for $v_{j}=\left.v\right|_{\Omega_{j}}$. Let $\zeta \in H^{m}(\Omega)$ be defined by $\left.\zeta\right|_{\Omega_{j}}=\zeta_{j}$ and $\tilde{v}=\Pi_{h} \zeta$. It follows from (2.6)-(2.8) that

$$
\operatorname{Tr}_{F} \tilde{v}=\operatorname{Tr}_{F} v \quad \forall F \in \mathcal{F}_{j}, 1 \leq j \leq J, \quad \text { and } \quad\|\tilde{v}\|_{H^{m}(\Omega)} \lesssim\|\zeta\|_{H^{m}(\Omega)} .
$$

By (1.1), (3.11) and Lemma 3.2, we have $a(v, v) \leq a(\tilde{v}, \tilde{v}) \lesssim\|\tilde{v}\|_{H^{m}(\Omega)}^{2}$. It then follows from (2.5) and (3.11) that

$$
\begin{aligned}
a(v, v) & \lesssim d^{1-2 m} \sum_{j=1}^{J} \sum_{|\alpha| \leq m} d^{2|\alpha|-1}\left\|\partial^{\alpha} \zeta\right\|_{L^{2}\left(\Omega_{j}\right)}^{2} \\
& \lesssim d^{1-2 m} \sum_{j=1}^{J} \sum_{|\alpha| \leq m-1} d^{2|\alpha|}\left[\left\|\partial^{\alpha} v\right\|_{L^{2}\left(\partial \Omega_{j}\right)}^{2}+d\left|\partial^{\alpha} v\right|_{H^{1 / 2}\left(\partial \Omega_{j}\right)}^{2}\right] .
\end{aligned}
$$

Since $h \lesssim d$, the estimate (3.10) follows from (1.2), (2.9) and (3.12).

The following lemma shows that (3.10) is the best possible estimate. 
Lemma 3.6. There exists $v_{*} \in V_{h}(\Gamma)$ such that

$$
h^{-2 m+1}\left(v_{*}, v_{*}\right)_{h, \Gamma} \lesssim a\left(v_{*}, v_{*}\right) .
$$

Proof. In view of (2.10), we may without loss of generality assume that there is a point $p$ in some $F \in \mathcal{F}_{j}$ such that the evaluation of $\partial_{n}^{m-1}$ at $p$ is one of the nodal variables of $V_{h}$. Let $v_{*} \in V_{h}(\Gamma)$ be defined by the property that $\left(\partial_{n}^{m-1} v_{*}\right)(p)=1$ and $N\left(v_{*}\right)=0$ for all other skeletal nodal variables $N$.

By (1.2) and a scaling argument we have

$$
\left(v_{*}, v_{*}\right)_{h, \Gamma} \approx h^{2 m+n-3} .
$$

On the other hand, (1.1), (2.4) and a scaling argument shows that

$$
a\left(v_{*}, v_{*}\right) \gtrsim\left|v_{*}\right|_{H^{m}(\Omega)}^{2} \gtrsim \sum_{j=1}^{J} \sum_{F \in \mathcal{F}_{j}} \sum_{|\alpha|=m-1}\left|\partial^{\alpha} v_{*}\right|_{H^{1 / 2}(F)}^{2} \gtrsim h^{n-2} .
$$

The estimate (3.13) follows from (3.14) and (3.15).

Corollary 3.7. We have $\lambda_{\max }\left(\mathbf{S}_{h}\right) \approx h^{-2 m+1}$.

Proof. This is an immediate consequence of (1.3), (3.10), (3.13) and the Rayleigh quotient formula (cf. [13])

$$
\lambda_{\max }\left(\mathbf{S}_{h}\right)=\max _{\substack{v \in V_{h}(\Gamma) \\ v \neq 0}} \frac{\left(\mathbf{S}_{h} v, v\right)_{h, \Gamma}}{(v, v)_{h, \Gamma}} .
$$

Combining Corollary 3.4 and Corollary 3.7, we have the following result.

Theorem 3.8. The estimate $\kappa\left(\mathbf{S}_{h}\right) \approx d^{-1} h^{-2 m+1}$ holds under the assumptions on the subdomains and the finite element space stated in Section 2.

\section{Extension to Nonconforming Finite Elements}

Let $W_{h} \subseteq L^{2}(\Omega)$ be a nonconforming finite element space (i.e., $W_{h} \nsubseteq H^{m}(\Omega)$ ) associated with $\mathcal{T}_{h}$. We assume that $\left.w\right|_{T} \in H^{m}(T)$ for all $w \in W_{h}$ and $T \in \mathcal{T}_{h}$. The variational form $a(\cdot, \cdot)$ cannot be directly applied to functions in $W_{h}$. Instead, we consider the bilinear form $a_{h}\left(w_{1}, w_{2}\right)=\sum_{T \in \mathcal{T}_{h}} a_{T}\left(w_{1}, w_{2}\right)$ for all $w_{1}, w_{2} \in W_{h}$, where $a_{T}(\cdot, \cdot)$ is the restriction to $T$ of the integral defining $a(\cdot, \cdot)$.

The analog of (1.1) for $W_{h}$ is

$$
a_{h}(w, w) \approx \sum_{T \in \mathcal{T}_{h}}\|w\|_{H^{m}(T)}^{2} \quad \forall w \in W_{h}
$$

Let $\mathcal{N}_{\Gamma}$ be the set of the nodal variables of $W_{h}$ on $\Gamma$. We define $W_{h}(\Omega \backslash \Gamma)$ to be the subspace $\left\{w \in W_{h}: \nu(w)=0 \forall \nu \in \mathcal{N}_{\Gamma}\right\}$ of $W_{h}$, and $W_{h}(\Gamma) \subseteq W_{h}$ to be the $a_{h}(\cdot, \cdot)$ orthogonal complement of $W_{h}(\Omega \backslash \Gamma)$. The functions in $W_{h}(\Gamma)$ are completely determined by the nodal variables in $\mathcal{N}_{\Gamma}$ and we can define an inner product $(\cdot, \cdot)_{h, \Gamma}$ on $W_{h}(\Gamma)$ by

$$
\left\langle w_{1}, w_{2}\right\rangle_{h, \Gamma}=h^{n-1} \sum_{\nu \in \mathcal{N}_{\Gamma}} h^{2|\nu|} \nu\left(w_{1}\right) \nu\left(w_{2}\right),
$$


where $|\nu|$ is the order of differentiation in the definition of the nodal variable $\nu$. The Schur complement operator $\mathbb{S}_{h}: W_{h}(\Gamma) \longrightarrow W_{h}(\Gamma)$ is then defined by

$$
\left\langle\mathbb{S}_{h} w_{1}, w_{2}\right\rangle_{h, \Gamma}=a_{h}\left(w_{1}, w_{2}\right) \quad \forall w_{1}, w_{2} \in W_{h}(\Gamma)
$$

Remark 4.1. We can define an inner product $((\cdot, \cdot))_{h, \Gamma}$ on a conforming $V_{h}(\Gamma)$ by

$$
\left(\left(v_{1}, v_{2}\right)\right)_{h, \Gamma}=h^{n-1} \sum_{\mu \in \mathcal{M}_{\Gamma}} h^{2|\mu|} \mu\left(v_{1}\right) \mu\left(v_{2}\right)
$$

where $\mathcal{M}_{\Gamma}$ is the set of nodal variables of $V_{h}$ on $\Gamma$. A scaling argument shows that

$$
(v, v)_{h, \Gamma} \approx((v, v))_{h, \Gamma} \quad \forall v \in V_{h}(\Gamma)
$$

We prefer to use the inner product $(\cdot, \cdot)_{h, \Gamma}$ in the conforming case because the definition (1.2) is simpler than (4.4).

Example 4.2. Some commonly used nonconforming finite elements for boundary value problems on $\mathbb{R}^{2}$ are the nonconforming $\mathcal{P}_{1}$ element (cf. [16] and Figure 8) for second order problems, the Adini element (cf. [1] and Figure 9) and the Morley element (cf. [19] and Figure 10) for fourth order problems.

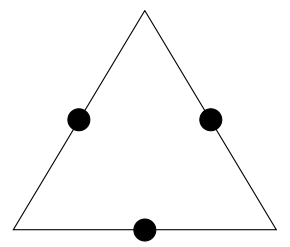

Figure 8

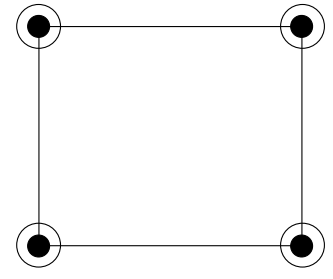

Figure 9

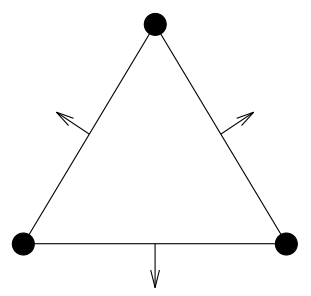

Figure 10

As in Examples 1.1 and 1.2, the condition numbers of the Schur complement operators $\mathbb{S}_{h}$ for these nonconforming finite elements are equivalent to the condition numbers of the Schur complement matrices obtained from the stiffness matrices.

Next we describe a framework that can derive condition number estimates for nonconforming Schur complement operators from the estimates for conforming finite elements. We make the following assumptions on the nonconforming finite element.

(A.1) The nodal variables of the nonconforming finite element include pointwise evaluation at points on the boundary of the element.

(A.2) There exists an interpolation operator $\pi_{h}: V \longrightarrow W_{h}$ such that

$$
\left[\sum_{T \in \mathcal{T}_{h}}\left|\zeta-\pi_{h} \zeta\right|_{H^{\ell}(T)}^{2}\right]^{1 / 2} \lesssim h^{m-\ell}|\zeta|_{H^{m}(\Omega)} \quad \forall \zeta \in V \quad \text { and } \quad 0 \leq \ell \leq m
$$


(A.3) There exists a conforming finite element space $V_{h}$ that has the properties described in Section 2 (cf. (2.7)-(2.10)), and there exists an operator $I_{h}: W_{h} \longrightarrow V_{h}$ such that

$$
\begin{array}{rlrl}
\left\|I_{h} w\right\|_{H^{m}(\Omega)}^{2} & \approx \sum_{T \in \mathcal{T}_{h}}\|w\|_{H^{m}(T)}^{2} & \forall w \in W_{h}, \\
\sum_{\mu \in \mathcal{M}_{\Gamma}} h^{2|\mu|}\left[\mu\left(I_{h} w\right)\right]^{2} \approx \sum_{\nu \in \mathcal{N}_{\Gamma}} h^{2|\nu|}[\nu(w)]^{2} & \forall w \in W_{h} .
\end{array}
$$

(A.4) There exists a point $p$ on some $F \in \mathcal{F}_{j}$ such that $w \rightarrow\left(\partial_{n}^{m-1} w\right)(p)$ belongs to $\mathcal{N}_{\Gamma}$ and $v \rightarrow\left(\partial_{n}^{m-1} v\right)(p)$ belongs to $\mathcal{M}_{\Gamma}$. Moreover, if $w \in W_{h}$ has the property that $\left(\partial_{n}^{m-1} w\right)(p)=1$ and $\nu(w)=0$ for any other $\nu \in \mathcal{N}_{\Gamma}$, then $\left(\partial_{n}^{m-1} I_{h} w\right)(p)=1$ and $\mu\left(I_{h} w\right)=0$ for any other $\mu \in \mathcal{M}_{\Gamma}$.

The following analog of Lemma 3.2 can be proved similarly.

Lemma 4.3. Suppose $w \in W_{h}(\Gamma), \tilde{w} \in W_{h}$ and $\nu(w)=\nu(\tilde{w})$ for all $\nu \in \mathcal{N}_{\Gamma}$. Then $a_{h}\left(w_{h}, w_{h}\right) \leq a_{h}(\tilde{w}, \tilde{w})$.

Theorem 4.4. The estimate $\kappa\left(\mathbb{S}_{h}\right) \approx d^{-1} h^{-2 m+1}$ holds under the assumptions (A.1)(A.4) on $W_{h}$ and the assumptions on the subdomains stated in Section 2.

Proof. By (1.1), (1.3), (4.1), (4.3)-(4.5), (4.7) and (4.8) we have

$$
\frac{\left(\mathbf{S}_{h} I_{h} w, I_{h} w\right)_{h, \Gamma}}{\left(I_{h} w, I_{h} w\right)_{h, \Gamma}} \approx \frac{\left\langle\mathbb{S}_{h} w, w\right\rangle_{h, \Gamma}}{\langle w, w\rangle_{h, \Gamma}} \quad \forall w \in W_{h}
$$

The Rayleigh quotient formulas and (4.9) imply that

$$
\lambda_{\min }\left(\mathbf{S}_{h}\right) \lesssim \lambda_{\min }\left(\mathbb{S}_{h}\right) \text { and } \lambda_{\max }\left(\mathbb{S}_{h}\right) \lesssim \lambda_{\max }\left(\mathbf{S}_{h}\right)
$$

Next we want to show that

$$
\lambda_{\min }\left(\mathbb{S}_{h}\right) \lesssim d
$$

Let $f$ be as in the proof of Lemma 3.3, $\tilde{w}=\pi_{h} f$ and $w_{*} \in W_{h}(\Gamma)$ be defined by

$$
\nu\left(w_{*}\right)=\nu(\tilde{w}) \quad \forall \nu \in \mathcal{N}_{\Gamma}
$$

It follows from (4.1), (4.6), (4.12) and Lemma 4.3 that

$$
a_{h}\left(w_{*}, w_{*}\right) \leq a_{h}(\tilde{w}, \tilde{w}) \lesssim\|f\|_{H^{m}(\Omega)}^{2}
$$

Note that (4.6) implies that polynomials of degree $\leq m-1$ are shape functions of the nonconforming finite element. Therefore $\tilde{w}$ is identically 1 on all triangles $T \in \mathcal{T}_{h}$ that are inside $\Omega_{\epsilon}$. We may also assume that $h \leq \epsilon$, otherwise (4.11) follows immediately from the 
second inequality in (4.10) and Corollary 3.7. This means that $\tilde{w}$ is identically 1 on $\Omega_{2 \epsilon}$. We obtain from (3.5), (4.2), (4.12) and (A.1)

$$
\left\langle w_{*}, w_{*}\right\rangle_{h, \Gamma} \geq h^{n-1} \sum_{\nu \in \mathcal{N}_{\Gamma},|\nu|=0}\left[\nu\left(w_{*}\right)\right]^{2} \gtrsim\left|\Gamma_{2 \epsilon}\right| \gtrsim d^{-1} .
$$

The two estimates (4.13) and (4.14) show that

$$
a_{h}\left(w_{*}, w_{*}\right) \lesssim d\left\langle w_{*}, w_{*}\right\rangle_{h, \Gamma}
$$

which together with (4.3) and the Rayleigh quotient formula yield the estimate (4.11).

Let $p \in F \in \mathcal{F}_{j}$ be the point stipulated in the assumption (A.4). Let $w_{\sharp} \in W_{h}(\Gamma)$ be defined by $\left(\partial_{n}^{m-1} w_{\sharp}\right)(p)=1$ and $\nu\left(w_{\sharp}\right)=0$ for any other $\nu \in \mathcal{N}_{\Gamma}$. According to the assumption (A.4), the function $\tilde{v}=I_{h} w_{\sharp}$ satisfies $\left(\partial_{n}^{m-1} \tilde{v}\right)(p)=1$ and $\mu(\tilde{v})=0$ for any other $\mu \in \mathcal{M}_{\Gamma}$. Let $v_{*} \in V_{h}(\Gamma)$ be defined by

$$
\mu\left(v_{*}\right)=\mu(\tilde{v}) \quad \forall \mu \in \mathcal{M}_{\Gamma} .
$$

The function $v_{*}$ coincides with the one defined in the proof of Lemma 3.6. By (1.1), Lemma 3.2, (3.13), (4.2), (4.4), (4.5), (4.7)-(4.8) and (4.16), we have

$$
a_{h}\left(w_{\sharp}, w_{\sharp}\right) \gtrsim a(\tilde{v}, \tilde{v}) \geq a\left(v_{*}, v_{*}\right) \gtrsim h^{-2 m+1}\left(v_{*}, v_{*}\right)_{h, \Gamma} \gtrsim h^{-2 m+1}\left\langle w_{\sharp}, w_{\sharp}\right\rangle_{h, \Gamma},
$$

which together with (4.3) and the Rayleigh quotient formula imply that

$$
\lambda_{\max }\left(\mathbb{S}_{h}\right) \gtrsim h^{-2 m+1}
$$

Combining Corollary 3.4, Corollary 3.7, (4.10), (4.11) and (4.18) we find $\lambda_{\min }\left(\mathbb{S}_{h}\right) \approx d$ and $\lambda_{\max }\left(\mathbb{S}_{h}\right) \approx h^{-2 m+1}$, and the theorem follows.

The framework described by assumptions (A.1)-(A.4) can be applied to the nonconforming finite elements in Example 4.2. It is clear that conditions (A.1)-(A.2) are satisfied by these elements. The space $V_{h}$ that appears in conditions (A.3)-(A.4) comes from a conforming relative of the nonconforming finite element.

For the nonconforming $\mathcal{P}_{1}$ element, we can take its conforming relative to be the $\mathcal{P}_{2}$ element. These elements are relatives in the sense that the shape functions of the $\mathcal{P}_{1}$ element are also shape functions of the $\mathcal{P}_{2}$ element and the nodal variables of the nonconforming $\mathcal{P}_{1}$ element are also nodal variables of the $\mathcal{P}_{2}$ element (cf. Figure 11).
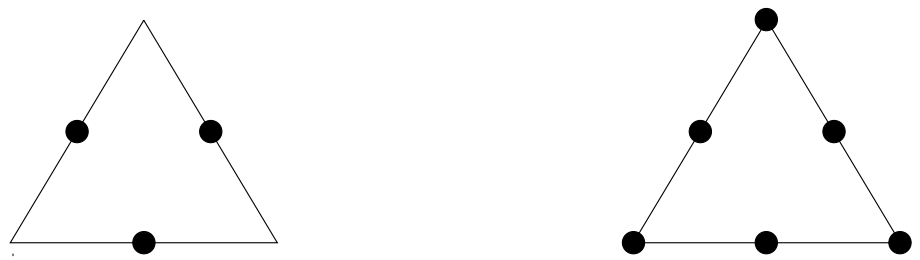

Figure 11 
Let $W_{h}$ be the nonconforming $\mathcal{P}_{1}$ finite element space and $V_{h}$ be the conforming $\mathcal{P}_{2}$ finite element space. We can construct $I_{h}: W_{h} \longrightarrow V_{h}$ as follows. Let $w \in W_{h}$. At a midpoint $m,\left(I_{h} w\right)(m)=w(m)$. At a vertex $p,\left(I_{h} w\right)(p)=w(m)$, where $m$ is an adjacent midpoint (i.e., both $p$ and $m$ belong to the same edge in $\mathcal{T}_{h}$ ). If $p \in \Gamma$, then we also choose $m$ from $\Gamma$.

For the Morley element, we can take its conforming relative to be the Hsieh-CloughTocher macro element (cf. Figure 10 and Figure 6). Let $W_{h}$ be the Morley finite element space and $V_{h}$ be the Hsieh-Clough-Tocher macro element space. We can construct $I_{h}$ : $W_{h} \longrightarrow V_{h}$ as follows. Let $w \in W_{h}$. Then $\left(\partial_{n} I_{h} w\right)(m)=\left(\partial_{n} w\right)(m)$ and $\left(I_{h} w\right)(p)=w(p)$ for any midpoint $m$ and any vertex $p$. Note that $\nabla w$ is also well-defined for $w \in W_{h}$, and we can define (at a vertex $p)\left[\nabla\left(I_{h} w\right)\right](p)=(\nabla w)(m)$, where $m$ is an adjacent midpoint. If $p \in \Gamma$, then we also choose $m$ from $\Gamma$.

A conforming relative for the Adini element is the Bogner-Fox-Schmit element (cf. Figure 9 and Figure 5). Let $W_{h}$ be the Adini finite element space and $V_{h}$ be the BognerFox-Schmit finite element space. The operator $I_{h}: W_{h} \longrightarrow V_{h}$ is defined as follows. Let $w \in W_{h}$ and $p$ be a vertex. Then $\left(I_{h} w\right)(p)=w(p),\left[\nabla\left(I_{h} w\right)\right](p)=(\nabla w)(p)$ and $\left(I_{h} w\right)_{x_{1} x_{2}}(p)=0$.

It is straightforward in all three cases to check that conditions $(\mathbf{A . 3})-(\mathbf{A} .4)$ are satisfied by $I_{h}$. We refer the readers to [6], [7] and [9] for some related estimates.

Therefore Theorem 4.4 can be applied to the nonconforming $\mathcal{P}_{1}$ finite element discretization of the Poisson equation and the Morley and Adini discretizations of the biharmonic and plate bending problems.

Remark 4.5. The framework developed in this section can of course be applied to many other nonconforming finite elements. For example, it is applicable to the Wilson elements (cf. [23]) for two- and three-dimensional elasticity problems, where we can take the relatives to be the two- and three-dimensional $\mathcal{Q}_{2}$ elements.

Acknowledgment The research in this paper began while the author was visiting the Institute for Mathematics and its Applications at the University of Minnesota. She would like to thank the IMA for their support and hospitality.

\section{References}

1. A. Adini and R.W. Clough, Analysis of plate bending by the finite element method, NSF Report G. 7337 (1961).

2. J.H. Argyris, I. Fried, and D.W. Scharpf, The TUBA family of plate elements for the matrix displacement method, Aero. J. Roy. Aero. Soc. 72 (1968), 701-709.

3. P.E. Bjørstad and O.B. Widlund, Iterative methods for the solution of elliptic problems on regions partitioned into substructures, SIAM J. Numer. Anal. 23 (1986), 1097-1120.

4. F.K. Bogner, R.L. Fox and L.A. Schmit, The generation of interelement compatible stiffness and mass matrices by the use of interpolation formulas, Proceedings of the Conference on Matrix Methods in Structural Mechanics (1965), Wright Patterson A.F.B., Ohio. 
5. J.H. Bramble and S.R. Hilbert, Estimation of linear functionals on Sobolev spaces with applications to Fourier transforms and spline interpolation, SIAM J. Numer. Anal. 7 (1970), 113-124.

6. S.C. Brenner, Two-level additive Schwarz preconditioners for nonconforming finite element methods, Math. Comp. 65 (1996), 897-921.

7., A two-level additive Schwarz preconditioner for nonconforming plate elements, Numer. Math. 72 (1996), 419-447.

8. S.C. Brenner and L.R. Scott, The Mathematical Theory of Finite Element Methods, Springer-Verlag, New York, 1994.

9. S.C. Brenner and L.-Y. Sung, Balancing domain decomposition for nonconforming plate elements, IMA Preprint Series, Number 1512, October 1997.

10. T.F. Chan and T.P. Mathew, Domain decomposition algorithms, Acta Numerica (1994), 61-143.

11. T.F. Chan, W. E and J. Sun, Domain decomposition interface preconditioners for fourth-order elliptic problems, Appl. Numer. Math. 8 (1991), 317-331.

12. P.G. Ciarlet, The Finite Element Method for Elliptic Problems, North Holland, Amsterdam, 1978.

13. Introduction to Numerical Linear Algebra and Optimization, Cambridge University Press, Cambridge, 1989.

14. P. Clément, Approximation by finite element functions using local regularization, R.A.I.R.O. R-2 (1975), 77-84.

15. R.W. Clough and J.L. Tocher, Finite element stiffness matrices for for analysis of plates in bending, Proceedings of the Conference on Matrix Methods in Structural Mechanics (1965), Wright Patterson A.F.B., Ohio.

16. M. Crouzeix and P.-A. Raviart, Conforming and nonconforming finite element methods for solving the stationary Stokes equations I, R.A.I.R.O. R-3 (1973), 33-75.

17. P. Grisvard, Elliptic Problems in Nonsmooth Domains, Pitman, Boston, 1985.

18. L. Mansfield, On the conjugate gradient solution of the Schur complement system obtained from domain decomposition, SIAM J. Numer. Anal. 27 (1990), 1612-1620.

19. L.S.D. Morley, The triangular equilibrium problem in the solution of plate bending problems, Aero. Quart. 19 (1968), 149-169.

20. L.R. Scott and S. Zhang, Finite element interpolation of nonsmooth functions satisfying boundary conditions, Math. Comp. 54 (1990), 483-493.

21. B. Smith, P. Bjørstad and W. Gropp, Domain Decomposition, Cambridge University Press, Cambridge, 1996.

22. H. Triebel, Interpolation Theory, Function Spaces, Differential Operators, North Holland, Amsterdam, 1978.

23. E.L. Wilson and R.L. Taylor, Incompatible displacement models, in Proceedings of the Symposium on Numerical and Computer Methods in Structural Engineering (O.N.R., University of Illinois, September, 1971). 